\title{
Bilateral necrotising scleritis with marginal corneal ulceration after cataract surgery in a patient with vasculitis
}

\author{
STEPHEN E. BLOOMFIELD, ${ }^{1}$ CARL G. BECKER, ${ }^{2}$ \\ CHARLES L. CHRISTIAN, ${ }^{3}$ AND JACK S. NAUHEIM ${ }^{4}$ \\ From the Departments of ${ }^{1}$ Ophthalmology and ${ }^{2}$ Pathology, New York Hospital-Cornell Medical Center, \\ the ${ }^{3}$ Department of Medicine, Hospital for Special Surgery, and the ${ }^{4}$ Department of Ophthalmology, \\ State University of New York at Stony Brook, USA
}

SUMMARY The onset of bilateral necrotising scleritis and marginal corneal ulceration after cataract surgery are described in a patient with diffuse vasculitis syndrome. The finding of immune complexes in this patient's tissue biopsy suggests that they are the cause of a localised primary vasculitis triggered by surgical inflammation. Clinically, this patient's marginal corneal ulceration preceded the scleral necrosis. A past history of orbital inflammation may have been due to a posterior scleritis. The corneal ulcerations responded to conjunctival debridement and steroids. The scleral necrosis was unresponsive to the 2 anti-inflammatory preparations tried, and the patient eventually went into remission while off all treatment.

Necrotising scleritis is the most serious and destructive form of scleral disease. It is rare and often associated with a variety of systemic disorders. This case report documents the onset of bilateral necrotising scleritis and marginal corneal ulceration after cataract surgery in a patient with a diffuse vasculitis syndrome. Immune complexes found in the conjunctiva and episcleral tissue may have been instrumental in triggering the inflammatory process.

\section{Case report}

A 67-year-old white female with a vasculitis syndrome presented for ophthalmological evaluation of painless, progressive loss of vision in both eyes. Her systemic disease began 3 years earlier with fever, chest pain, anaemia, and evidence of renal failure. A renal biopsy showed 'focal lobular glomerulitis'. Early in the course of her illness chronic serous otitis of the right ear required treatment with a Teflon tube tympanectomy. Methylprednisone therapy was instituted and maintained continuously. The association of a vasculitis syndrome after acute otitis media has been reported. ${ }^{1}$ At the time of ophthalmological consultation

Correspondence to Stephen E. Bloomfield, MD, 525 East 68th Street, New York, New York 10021, USA.
(March 1977) the dose of methylprednisone was $48 \mathrm{mg}$ and $32 \mathrm{mg}$ on alternate days.

Two years before this eye examination she had what was diagnosed as a left orbital inflammation, which resolved without sequelae on systemic corticosteroids. That episode started with a limitation of elevation of the globe and was accompanied by lid oedema and chemosis. An ultrasonogram at that time revealed thickening of the extraocular muscles.

Her general physical examination when first seen at the New York Hospital-Cornell Medical Center showed a thin, frail-appearing woman confined to a wheelchair because of severe weakness and pain due to fractures of the left pubic ramus. Her blood pressure was $160 / 90 \mathrm{mmHg}$, there were ecchymotic skin lesions over the extremities, and she had a mild left hemiparesis. Notable laboratory data were: haematocrit 23; serum creatinine $2.2 \mathrm{mg} / 100 \mathrm{ml}(194 \mu \mathrm{mol} / \mathrm{l})$ a trace of protein in the urine; latex fixation test negative; hepatitis $B$ surface antigen negative.

Ocular examination showed a best corrected visual acuity of counting fingers at $5 \mathrm{ft}(1.5 \mathrm{~m})$ on the right and hand motions on the left. Schirmer testing showed an adequate tear volume in both eyes, though slit-lamp examination revealed some filament formation on the left cornea, suggestive 


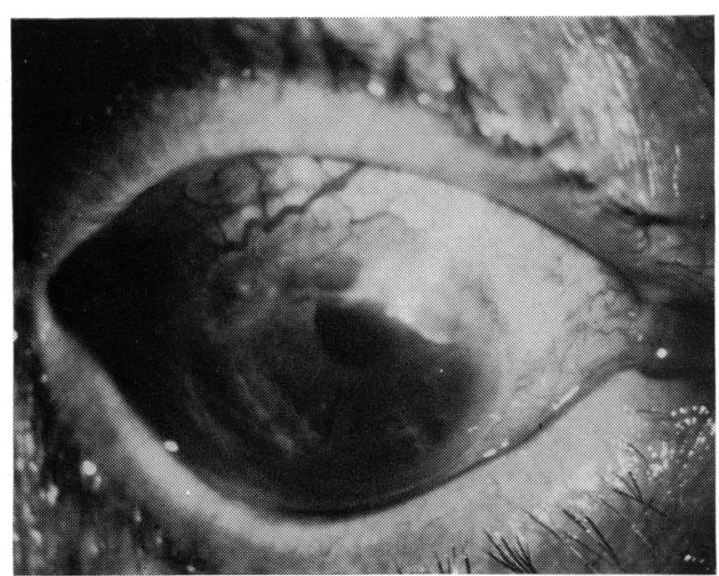

$1 \mathrm{~A}$

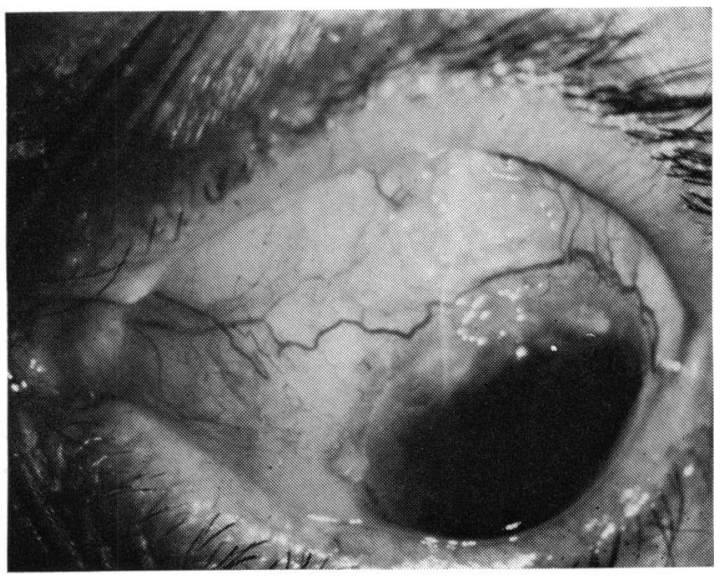

1B

Fig. 1A Marginal corneal ulceration and infiltration of the right eye. B: Similar corneal ulceration and early scleral necrosis of the left eye. There is prominent vessel formation in the limbal area.

of a tear volume deficit. There was no corneal infiltration or inflammation of the anterior chamber. There were dense lens opacities in both eyes, the left greater than the right. There was no sign of present or past scleral inflammation. The intraocular pressure was $17 \mathrm{mmHg}$ in each eye. Indirect ophthalmoscopy through the dense lens opacities revealed a normal disc, vessels, and macular area in each eye.

The patient underwent an uncomplicated left intracapsular lens extraction on 18 May 1977. The wound was closed with 5 buried 8-0 silk sutures beneath a limbal flap. She had an uncomplicated postoperative course and was maintained on atropine $1 \%$ drops 4 times a day, chloramphenicol drops 4 times a day, and dexamethasone phosphate drops twice a day for 5 weeks. The second or right eye underwent a similar uncomplicated cataract extraction 6 weeks after the first operation. The same technique with silk sutures was used. Three months after her last operation she was comfortable and seeing 20/25 in both eyes from her aphakic spectacles. The eyes were uninflamed except for some injection at each limbal area.

She returned 3 weeks later complaining of discomfort in both eyes. Examination of the right eye revealed erosion of a suture at the 11 o'clock position $1 \mathrm{~mm}$ from the limbus. There was also some peripheral corneal infiltrate from the 11-1 o'clock position. Examination of the left eye showed only erosion of a suture through the conjunctiva at the 10 o'clock position of the limbus. The superior limbal conjunctiva of each eye appeared somewhat oedematous. We thought we might be dealing with a peripheral staphylococcus corneal infection and/or a suture abscess. The exposed sutures were removed and topical chloramphenicol treatment was instituted. Culture of each eye showed only a few colonies of Staphylococcus epidermidis.

One week later the patient returned with more corneal infiltrate on the right and the start of infiltrate on the left. Reculture showed no organisms. She was started on topical dexamethasone every 2 hours. A week later she returned still very uncomfortable, with the process of peripheral corneal infiltration continuing. The limbal conjunctiva looked more inflamed and oedematous. The remaining buried sutures were removed in the office at the slit-lamp, and the patient continued on topical corticosteroids every 2 hours while awake and chloramphenicol eye drops 4 times a day. The remaining sutures were removed because it was thought they might be playing a role in the continuation of this uncontrolled inflammation. She returned 5 days later with more peripheral corneal infiltrate, loss of corneal stroma in each eye, and scleral melting on the left, radiating from the original 10 o'clock suture tract (Figs. 1A and B).

At this time we decided to debride the oedematous, inflamed, and necrotic-appearing limbal conjunctiva for therapeutic and diagnostic purposes. The patient was admitted to the New York Hospital and taken to the operating room. The friable conjunctiva and episcleral tissue over each limba area were surgically resected in the form of an ellipse concentric to the limbus. The superior $180^{\circ}$ from the limbus to $4 \mathrm{~mm}$ posterior was removed in each eye. The tissue was placed in appropriate media and sent for histological and immuno- 


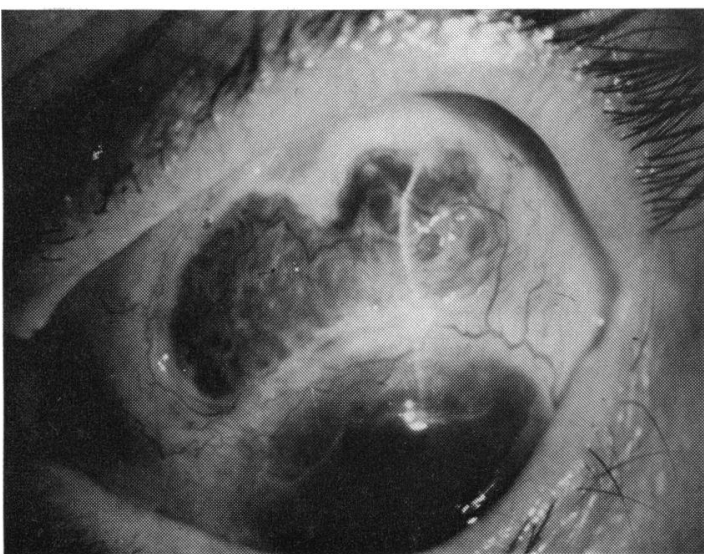

$2 \mathrm{~A}$

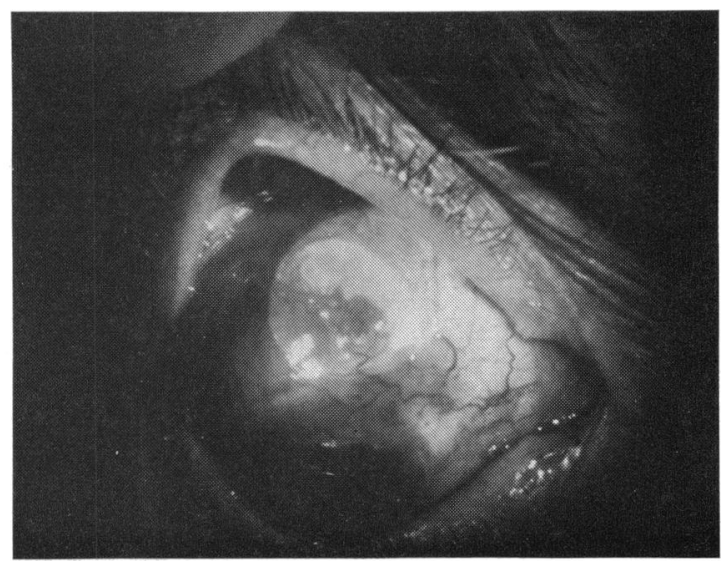

$2 B$

Fig. 2A Advanced scleral necros; with healing of the marginal corneal ulcerations in the left eye. A characteristically white avascular rim of necrosis is present. B: Fluorescein stain shows residual area of scleral necrosis in the right eye.

fluorescent studies. Postoperatively the patient was placed on hourly topical dexamethasone in each eye around the clock for 1 week, which was gradually tapered off as the corneal infiltrate disappeared. Three weeks after removal of the conjunctiva the corneas cleared of infiltrate, developed epithelium, and the patient became much more comfortable. The scleral melting continued in the left eye insidiously (Fig. 2A).

It was difficult to convince the patient that she still had a serious problem, since she was now comfortable and the eyes were less injected. She was started on oral indomethacin, $100 \mathrm{mg}$ per day in divided doses, to control the scleral necrosis. The topical corticosteroids were continued once a day in each eye. The scleral necrosis continued. After 1 month the patient was started on oxyphenbutazone, $600 \mathrm{mg}$ per day in divided doses. The scleral necrosis continued on the left and became worse on the right. This medication was discontinued when no effect was shown after 3 weeks. No other medication was given at this time because of a drop in the patient's platelet count. The scleral necrosis continued for 1 month while the patient was off all medication and then suddenly stopped in the left eye and slowed in the right (Fig. 2B). She has now been stable for the last 6 months.

\section{HISTOPATHOLOGICAL FINDINGS}

Microscopically the arteries, arterioles, and veins in the conjunctival-episcleral biopsy were surrounded by a cellular infiltrate consisting of polymorphonuclear neutrophils, lymphocytes, eosinophils, and in some areas plasma cells. The walls of some vessels were extensively infiltrated by inflammatory cells (Figs. 3A and B). In addition, margination and diapedesis of polymorphonuclear neutrophils were present.

Frozen sections of the biopsy which had been embedded in gelatin were treated with fluoresceinated rabbit antisera to human IgG, IgA, IgM, IgE, C3, and fibrinogen. Examination of these sections revealed focal granula deposits of $\mathrm{IgG}$ (Fig. 3C) IgM, and C3 (Fig. 3D) in the walls of conjunctival vessels, indicating that immune complexes had been deposited. In addition fibrin was deposited focally in vessel walls, presumably at sites of injury (Fig. 3E).

\section{Discussion}

Necrotising anterior scleritis is a rare and destructive disease resulting in necrosis and disappearance of the scleral envelope of the eye. ${ }^{2}$ Its occurrence after cataract extraction is unusual. The finding of immune complexes in the inflamed tissue suggests that they are the cause of localised vasculitis in this patient.

In the biopsied conjunctival tissue of this patient polymorphonuclear neutrophils and eosinophils were adherent to the endothelium of many vessels and were seen between endothelial cells, presumably attempting to phagocytose immune complexes deposited in the lining and in the walls of these vessels. It has been shown that, when polymorphonuclear leucocytes attempt to ingest immune complexes on nonphagocytosable surfaces such as vascular membranes, lysosomal enzymes are released into the surrounding tissues. ${ }^{34}$

The question arises as to why immune complexes 


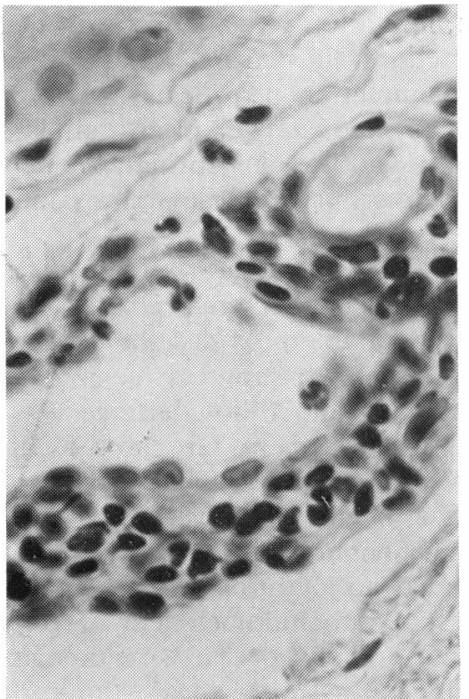

$3 \mathrm{~A}$

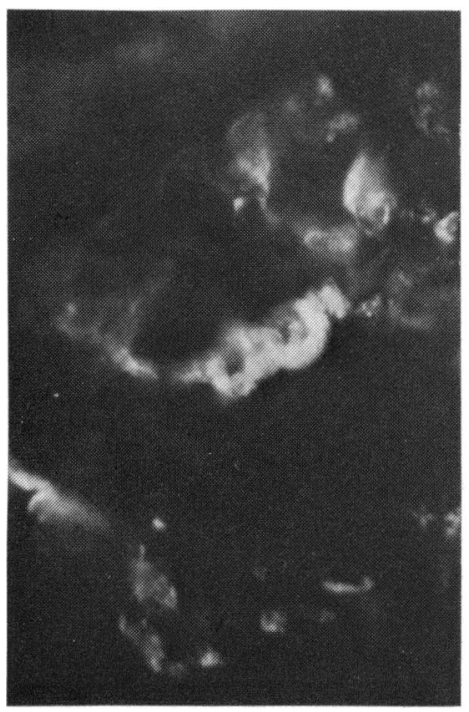

3D

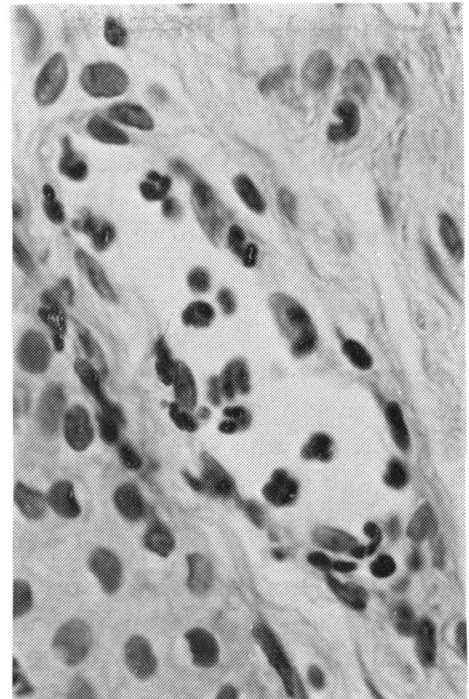

3B

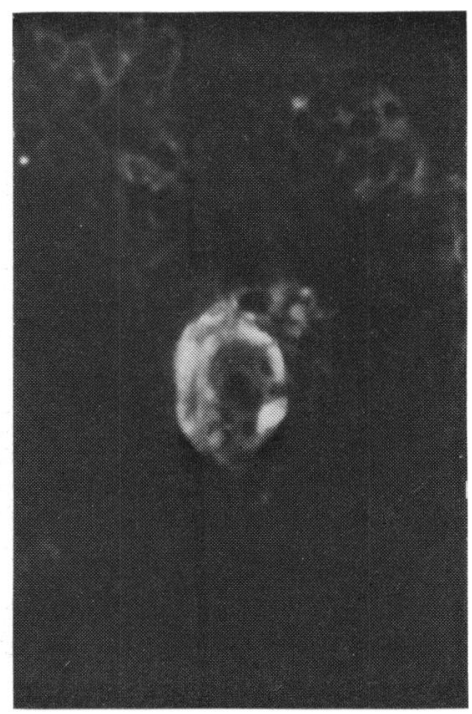

$3 \mathrm{E}$

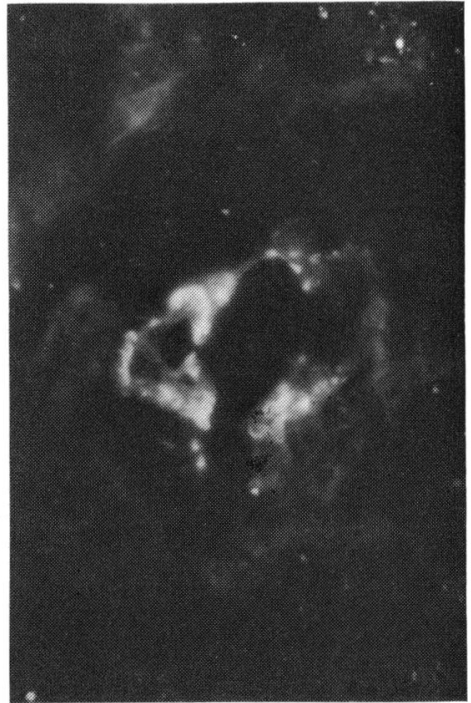

$3 C$

Fig. 3A The wall of the small vein is infiltrated by inflammatory cells. Polymorphonuclear neutrophils are adherent to the endothelium.

Haemaioxyiin and eosin. $\times 385$. B: Polymorphonuclear leucocytes are adherent to the endothelium of a venule and can be seen emigrating between endothelial cells. Haematoxylin and eosin. $\times 385$. C: Frozen section of conjunctival biopsy treated with fluoresceinated rabbit antiserum to $\mathrm{IgG}$. Granular immunofluorescent deposits of IgG are present in the wall of a small blood vessel. $\times 306$. D: Frozen section of conjunctival biopsy treated with fluoresceinated rabbit antiserum to human C3. Granular immunofluorescent deposits of $\mathrm{C} 3$ are present in the walls of a small blood vessel. $\times 306$. E: Frozen section of conjunctival biopsy treated with fluoresceinated antiserum to human fibrinogen. The wall of an injured vessel is insulated by fibrin. $\times 306$. were deposited in conjunctival-episcleral vessels in this patient, who at this time had no clinical evidence of immune complex deposition in other vascular beds, such as renal glomeruli. We have no answer to this, but we can offer 2 hypotheses.

The first hypothesis concerns the nature of the blood vessels of the external eye in this patient. Corneal and scleral necrosis began after the patient underwent surgery for cataracts. New vessels proliferated during healing of the wound occurred, and it is in this new area that immune complexes were deposited (Fig. 2B). This may indicate that new vessels are more susceptible to deposition of circulating immune complexes. If this is true, it would explain the progressive nature of the disease, since a situation would be created in which injury would follow reparative vessel growth as long as immune complexes remained in the circulation. According to this hypothesis patients with circulating immune complexes would be at particular risk of developing the scleral necrosis syndrome, not because of defective healing but because of increased vulnerability of new vessels.

The second hypothesis, which is not exclusive 
of the first, concerns the nature of the patient's circulating immune complexes. Although we could not demonstrate cryoglobulinaemia during the period of her illness described above, cryoglobulinaemia had been demonstrated in the past during periods of clinically active systemic disease. Cryoglobulin complexes might be expected to be deposited selectively in superficial vessels of the external eye, where the temperature would be lower than in vessels of internal organs. The two hypotheses together suggest the progression of scleral necrosis in this patient may have been a result of synergy between the increased susceptibility of new blood vessels to immune complex deposition and the thermal optimum for precipitation of cryoglobulins.

This hypothesis also suggests that patients with a history of rheumatoid arthritis, lupus erythematosus, or other diseases associated with circulating immune complexes might be at a special risk of developing scleral melting after ophthalmic surgery and should be examined for the presence of circulating complexes by a variety of techniques now available.

Our patient presented initially with discomfort and signs of marginal corneal ulceration. With resolution of the corneal involvement the symptoms subsided, but the destructive scleral process continued in an insidious manner, revealing the correct diagnosis of necrotising scleritis.

The corneal changes are similar to a Mooren's ulcer, which one might consider in the diagnosis in the absence of scleral involvement. Both types of ulcers are painful and gradually progressive. Mooren's ulceration, however, is not associated with any systemic disease or progressive scleral ulceration. ${ }^{5}$

The patient's past episode of left orbital cellulitis, presenting first with a limitation of elevation, may have been due to an unrecognised posterior scleritis. Inflammation of the posterior sclera is a difficult diagnosis to make and is deduced from its secondary effects on neighbouring tissues. The patient presents with pain, limitation of ocular movements, chemosis, and sometimes lid oedema. The indirect consequence of inflammation provides the clue to diagnosis, but its appearance will often simulate orbital cellulitis or pseudotumour formation. Bertelsen $^{6}$ confesses to this mistake in diagnosis in 6 out of 12 patients.

The treatment of deep scleritis at various stages is controversial. Watson and Hayreh ${ }^{2}$ believe that scleritis is medically treatable provided the dosages of the drugs are adequate to suppress the inflammatory change. Aronson and Elliot disagree.

Many anti-inflammatory agents are now available, but only a few are effective in the treatment of scleral disease. These include corticosteroids, indomethacin, and oxyphenbutazone. Some difficult cases have responded to cytotoxic agents. ${ }^{8}$ Our patient did not respond to indomethacin or oxyphenbutazone. She underwent a spontaneous remission after being off systemic medications for 1 month. The controversy surrounding the treatment of deep scleritis is probably due to its unpredictable course.

\section{References}

'Sargent JS, Christian CL. Necrotising vasculitis after acute serous otitis media. Ann Intern Med 1974; 81: 195-9. ${ }^{2}$ Watson PG, Hayreh SS. Scleritis and episcleritis. $B r J$ Ophthalmol 1976; 60: 163-91.

${ }^{3}$ Henson PM, Oades ZG. Stimulation of human neutrophils by soluble and insoluble immunoglobulin aggregates. J. Clin Invest 1975; 56: 1053-61.

${ }^{4}$ Henson PM. In: Bayer Symposium VI. Experimental Models of Chronic Inflammatory Diseases. New York: Springer, 1977; 94-106.

${ }^{5}$ Ferry AP, Leopold IH. Marginal corneal ulcer as a presenting manifestation of Wegener's granuloma. Trans Am Acad Ophthalmol Otolaryngol 1970; 74: 1276-82.

${ }^{6}$ Bertelsen TI. Acute sclerotenonitis and ocular myositis complicated by papillitis and retinal detachment and glaucoma. Acta Ophthalmol 1960; 38: 136-52.

${ }^{7}$ Aronson SB, Elliot JH. Scleritis. In: Golden B, ed. Ocular Inflammatory Disease. Springfield: Thomas, 1974; 43.

${ }^{8} \mathrm{Jampol}$ LM, West C, Goldberg MF. Therapy of scleritis with cytotoxic agents. Am J Ophthalmol 1978; 86: 266-71. 